\title{
OPTIMIZATION OFSPIRAL INDUCTOR WITH DIFFERENT INNER DIAMETERS
}

\author{
Beeresha R S $\mathbf{1}^{1}$, A M Khan ${ }^{2}$, Manjunath Reddy H V $\mathbf{V}^{3}$ \\ ${ }^{1}$ Research Scholar, Department of Electronics, Mangalore University, Karnataka, India \\ ${ }^{2}$ Professors, Department of Electronics, Mangalore University, Karnataka, India \\ ${ }^{3}$ General Manager, RF\& Microwave Design, ICON Design Automation Ltd, Karnataka India
}

\begin{abstract}
A scalable and highly accurate RF/Microwave symmetricalinductor has been designed using microstrip line. The 18 circular and 18 square structures are optimized with $600 \mathrm{MHz}$ operating frequency. The characteristic interest on high frequency operating circuits is inductance value and quality factor of the spiral inductor. The value of the spiral inductor is depends on conductor width $(W)$ space between the conductor $(S)$, turns $(n)$ outer diameter and inner diameter of the spiral inductor. In this design change the distribution of an inner diameter from 5mil to 10milfor 3 to 5 turns of spiral inductors. The optimization of inner diameter distribution is done by keeping space (1mil)between the conductor and width (2mil) of the conductor as constant. The changing inner diameter will effect to change in magnitude of the inductance and quality factor due passive capacitive and inductive reactance effect. These spiral inductors are designed using aluminum (Al) substrate material with $15 \mathrm{mil}$ height from the ground plane. The cupper material is used as a conductor of thickness $0.302 \mathrm{~mm}$. The aim of this paper is to compare the electromagnetic simulated results with simple equations of modified wheeler (MW), current sheet Approximation (CSA) and monomial equation $(M E)$. The passive component effect the spiral inductor is examines the variation of inductance and quality factor. The observed electromagnetic field simulated results gives better agreement with standard simple mentioned equations with $600 \mathrm{MHz}$ operating frequency. The validation of spiral inductor is done with different inner diameter (5mil to 10mil) and number of turns (3turns to 5 turns) of the spiral inductor.
\end{abstract}

Keywords: Quality Factor, Microstrip, Spiral Inductor, Radio Frequency, S-parameter, SRF.

\section{INTRODUCTION}

The communication system use considerable amount of passive components for design circuits like power amplifiers, oscillators, microwave switches, combiners, and splitters, etc. There have been numerous publications on spiral inductor design at microwave frequencies [1-5]. The implementation of integrated inductors has been great challenge in designing high frequency applications.

The on-chip spiral inductors have become importance by increasing frequency of the circuit. The passive components are major part in the circuit by referring Ericsson cellphone resulting that, in a cell phone board, the passive devices use to account $95 \%$ of the total component count, $80 \%$ of the board area and $70 \%$ of the board assembly cost [13]. The passive and active component ratio in the cell phone board is $21: 1$ [14].

The conventional computer aided tools are used to design fast and accurate modeling of the spiral inductors in $\mathrm{RF} /$ microwave applications. The on chip spiral inductor suffers from parasitic effect and accuracy is low. It needs to design suitable accurate multilayer integrated circuits by selecting suitable substrate material along with geometric parameters (width, space and inner diameter)of the models. The different circuit level models have been proposed by the researchers [6-12].
The operating frequency of the circuit increases but difficult to achieve better quality factor. The efficiency of spiral inductor is depending on quality factor of the signal. The researchers have been design using suitable inductor model by using substrate material and geometric calculation to improve the low quality factor of the inductor [16-18].

In this paper design symmetrical circular and square shaped spiral inductor and electromagnetic simulated field using NI/AWR (National Instrumentation/Applied Wave Research) tool. The obtained results are compared with MW, CSA and MEsimple equation and have good agreement with designed inductance value. The obtained spiral inductorresults gives better quality factors at $600 \mathrm{MHz}$ operating frequency.

The paper is organized as follows; Section II presents the basic of the spiral inductor design with equivalent circuit. In section III the geometric description of the circular and square spiral inductor with different inner diameter and turns. In section IV, the electromagnetic simulated results of different structures are compared and discussed with the help of observed results. In section $\mathrm{V}$ we summarize the obtained results.

\section{BASICS OF SPIRAL INDUCTOR}

The characteristics of the spiral inductor usually having three important figure of merit like inductance, quality 
factor and self-inductance (SRF) [19]. The physical designed parameters are the width of the conductor $(\mathrm{W})$, space between turns $(S)$, number of turns $(n)$, outer diameter $\left(D_{\text {out }}\right)$ and inner diameter $\left(D_{\text {in }}\right)$ which determines the value of the inductance and quality factor of inductor. All these physical parameters are designed using basic reference equations $[20,21]$.

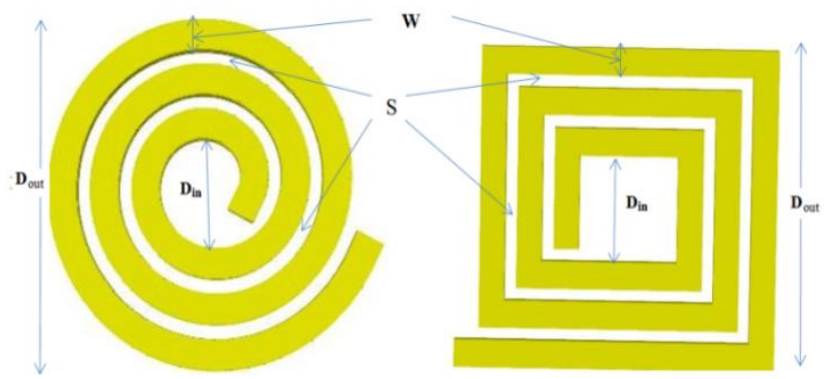

Fig-1: Circular and Square Spiral Inductor Structure

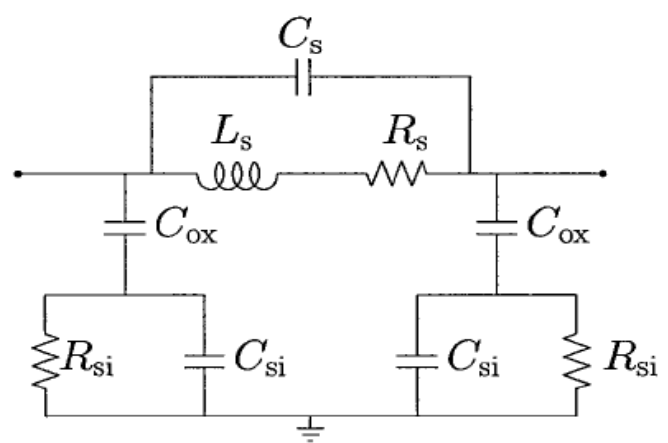

Fig-2: Equivalent Circuit of Spiral Inductors

The frequency dependency of the spiral metal coils series inductance and resistance are represented by the parallel $R_{S}$ and $\mathrm{L}_{\mathrm{s}}$ of the circuit. The $\mathrm{C}_{\mathrm{s}}$ is the series capacitance of the conductor and $\mathrm{C}_{\mathrm{ox}}$ is present in space between parallel conductors. The $\mathrm{R}_{\mathrm{si}}$ and $\mathrm{C}_{\mathrm{si}}$ represent the $\mathrm{DC}$ resistance of the metal line [22]. To facilitate the design of such components, significant work has gone into modeling spiral inductors using lumped circuit models [23], [24]. The parasitic resistors and capacitors in this model have simple physically intuitive expressions, but the inductance value lacks a simple but accurate expression.

The figure 2 shows the equivalent circuit for planar spiral inductor. Using this equivalent circuit to calculate inductance value for given frequency with the help of scattering parameters ( $\mathrm{S}$-parameter). The $\mathrm{S}$-parameters are calculated in AWR tool and transformed in to Y parameters from which the inductance $\mathrm{L}$ and $\mathrm{Q}$ factor can be calculated based on following equations [25-26]

$$
L=\frac{\operatorname{Im}\left(\frac{1}{Y_{21}}\right)}{2 \pi f}
$$

The quality factor can be generally expressed as:

$$
Q=\frac{i m\left(\frac{1}{Y}\right)}{\operatorname{Re}\left(\frac{1}{Y}\right)}
$$

Where $\mathrm{Y}$ : are the $\mathrm{Y}$ parameter and $\mathrm{f}$ is a signal frequency. The SRF for an inductor can be viewed as the maximum frequency at which the inductor value is positive. When the frequency increases, the inductor value becomes negative, i.e., the inductor acts like a capacitor.

\section{DESIGN SPIRAL INDUCTOR}

In this section discuss the analytical optimization of the spiral inductor using equations is performed, and the effect of design parameters on inductance, quality factor and selfresonant frequency (SRF). It should benoted that optimization is based on changing one parameter, while the other parameters are constants. In this paper we consider inner diameter as variable parameter.

To determine the optimum inductor parameters through optimization of MW, CSA and MEexpressions with electromagnetic field simulated results. In section discuss these methods with relative parameters[27].

\subsection{Modified Wheeler (Mw) Formula}

The wheeler presented several formulas for planar spiralinductors to calculate inductance in circular, square structure [28].The MW formula has found that a simple modification of the original Wheelerformula allows us to obtain an expression that is valid forplanar spiral integrated inductors.

$$
L_{M W}=K_{1} \mu_{0} \frac{n^{2} d_{\text {avg }}}{1+K_{2} \rho}
$$

Where $\rho$ is the fill ratio, $K_{1}$ and $K_{2}$ are integral constants and are layout dependent [27]. The $\mathrm{d}_{\mathrm{avg}}$ is average difference between outer and inner diameter of a spiral inductor.

\subsection{Current Sheet Approximation (Csa)}

The CSA is one of the simple expressions to find the inductance by approximating the sides of the spiral inductor [29]. This method is used to analyze self and mutual inductance by considering the opposite parallel sides of the spiral inductor. The self and mutual inductance is evaluated using the concept of geometric mean distance (GMD) [27].

$$
L_{g m d}=\frac{\mu n^{2} d_{\text {avg }} C_{1}}{2}\left(\ln \left(\frac{C_{2}}{\rho}\right)+C_{3} \rho+C_{4} \rho^{2}\right)
$$

Where the confidents of $C_{i}$ are layout dependent, $\mu$ is permeability of a substrate, $\mathrm{d}_{\mathrm{avg}}$ is equal to average distance between inner diameters $\left(D_{i}\right)$ to outer diameter $\left(D_{\text {out }}\right)$ and $\rho$ is the fill ratio.

\subsection{Monomial Expression (Me)}

The ME is one of the simple expressions to find the inductance value using data fitting technique in planar spiral inductor.

$$
C_{\text {mon }}=\beta d_{\text {out }}^{\alpha_{1}} W^{\alpha_{2}} d_{\text {avg }}^{\alpha_{3}} n^{\alpha_{4}} S^{\alpha_{5}}
$$


Where $\beta$ and $\alpha_{i}$ are layout dependents and $\mathrm{d}_{\text {out }}, \mathrm{W}, \mathrm{d}_{\mathrm{avg}}, \mathrm{n}$ and $\mathrm{S}$ are monomial variables of the spiral inductor.

The monomial expression is useful like other expressions foraccurate and simple to calculation of inductor value. It can be used for optimal design of spiral inductors geometric design $[27,30]$.

The spiral circular and square inductors are designed using physical parameters with the help of equivalent model. The aluminum $(\mathrm{Al})$ substrate material is used with $15 \mathrm{mil}$ above from the ground plane and it is suitable for $600 \mathrm{MHz}$ operating frequency. The spiral inductor is analyzefor different turns ( 3 turns to 5 turns) with inner diameters from $5 \mathrm{mil}$ to $10 \mathrm{mil}$ for operating frequency of $600 \mathrm{MHz}$. The obtained result is compared with these expression are well match with each other. The electromagnetic field simulated results and quality factor is discussed in below section.

\section{RESULTS AND DISCUSSION}

The symmetrical microstrip spiral inductors of circular and square structure are electromagnetic field simulated using NI/AWR tool. The design of spiral inductor with the help multilayer microwave integrated circuit (MMIC) technology. The aluminum substrate material is used at $15 \mathrm{mil}$ height from the ground plane and copper with $0.0305 \mathrm{~mm}$ thickness.

The symmetrical spiral inductors design and observe inductance $600 \mathrm{GHz}$ for 3 to 5 turns and inner diameter of $5 \mathrm{mil}$ to $10 \mathrm{mil}$. The quality factor of the spiral inductor is also studied for different inner diameters and turns. The simulated results of inductance different turns and inner diameter presented in figure 5, 7,9, 11, 13 and 15. It is reasonable to have higher quality factor and decrease in inductance with increase in turn width of the spiral inductor, which is also proved using electromagnetic field Simulated results. From the results in Fig. 6, 8, 10, 12, 14 and 16shows the spiral inductors higher Quality factor, but degradation in inductance as compared to variable inner diameter.

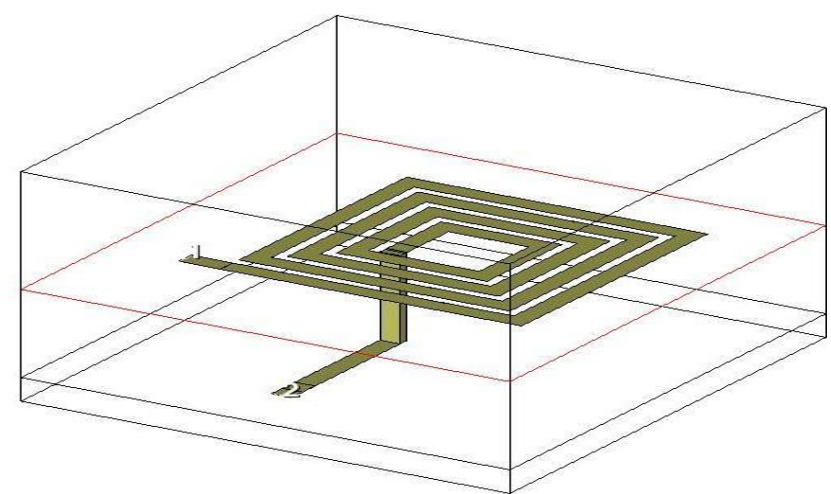

Fig-3: 3D Structure of 4 turns Spiral Inductor

The figure 3 shows one of the square 3D mess structures with three layers. The figure 4 shows current simulated structure of the above 3D mess structure example of 4 turns with inner diameter of $8 \mathrm{mil}$. The input is applied to second layer and drawn out from third layer. This construction is done by using NI/AWR tool. Similarly 3 to 5 turn spiral inductors are simulated and plot the graph frequency verses inductance and quality factor. The quality factor magnitude is considered for desired operating frequency of $600 \mathrm{MHz}$.

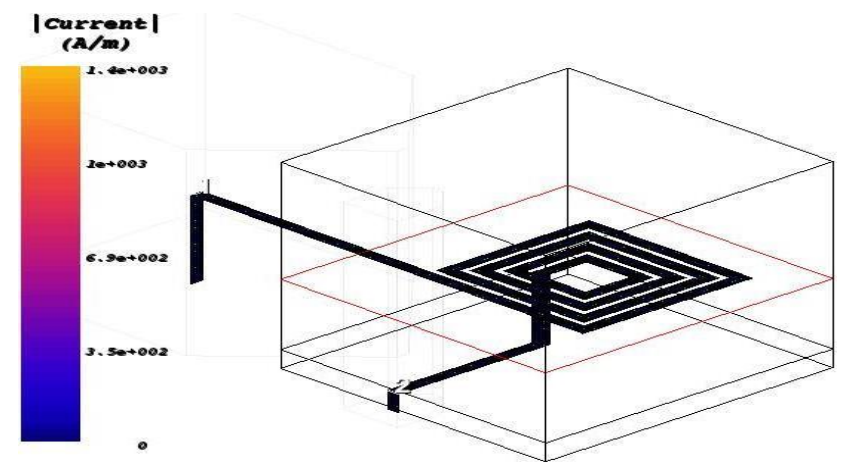

Fig-4:3D Current Simulated Structure

In a symmetrical structure, the most of mutual inductance effect occurs towards the center of the spiral inductor. The variation of the inner diameter length will affect to change total inductance due to change outer diameter of the spiral inductor. The magnitude of the quality factor is measured and analyzed using scattering parameter technique. The below plotted graphs shows the electromagnetic field simulated results of inductance and quality factors with respect to $600 \mathrm{MHz}$.

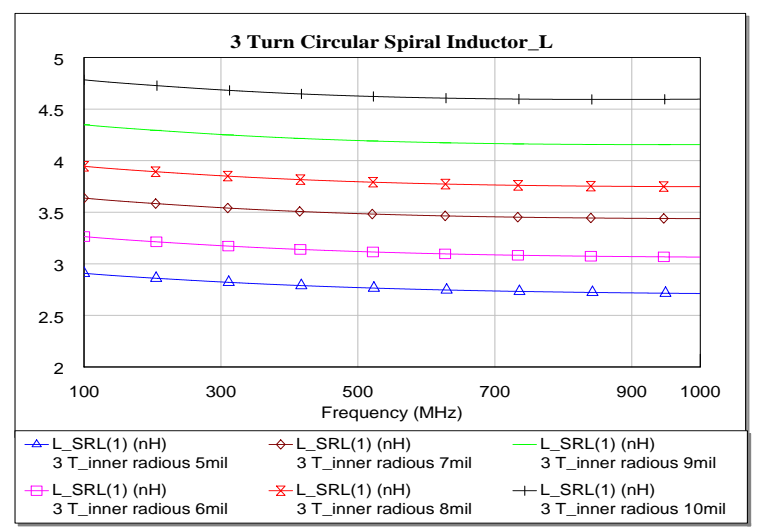

Fig-5: 3 Turns Circular Spiral Inductor (L)

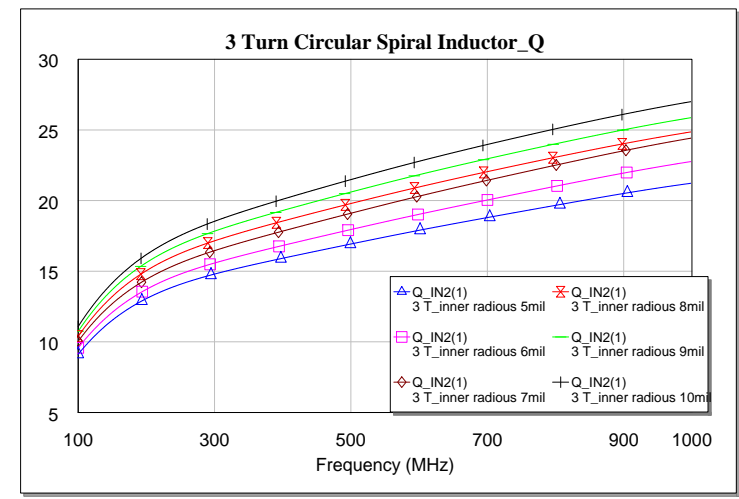

Fig-6: 3 Turns Circular Spiral Inductor (QF) 


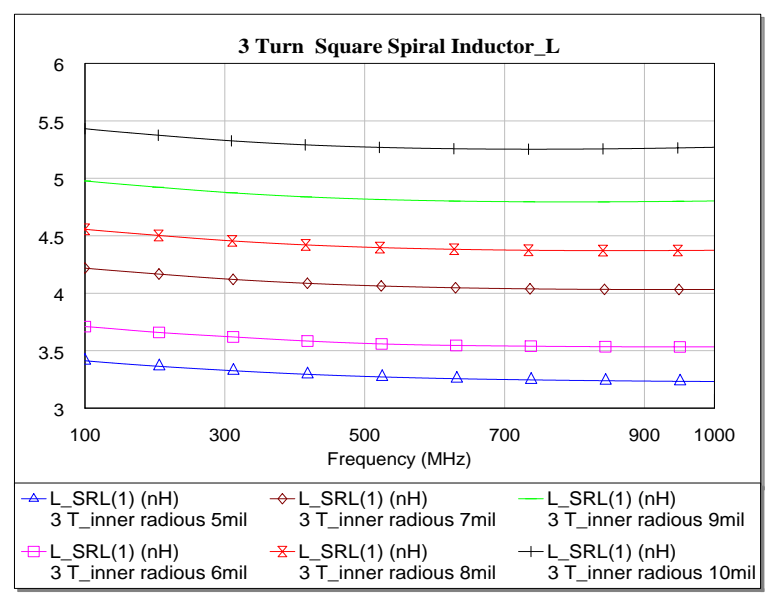

Fig-7: 3 TurnsSquare Spiral Inductor (L)

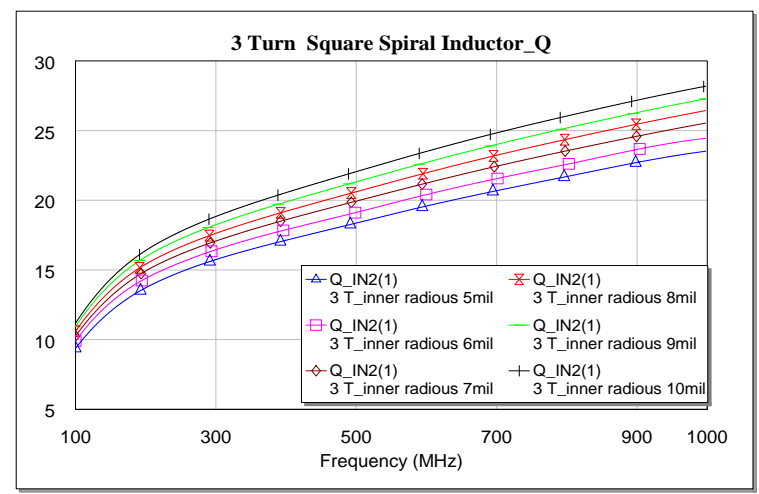

Fig-8: 3 TurnsSquare Spiral Inductor (QF)

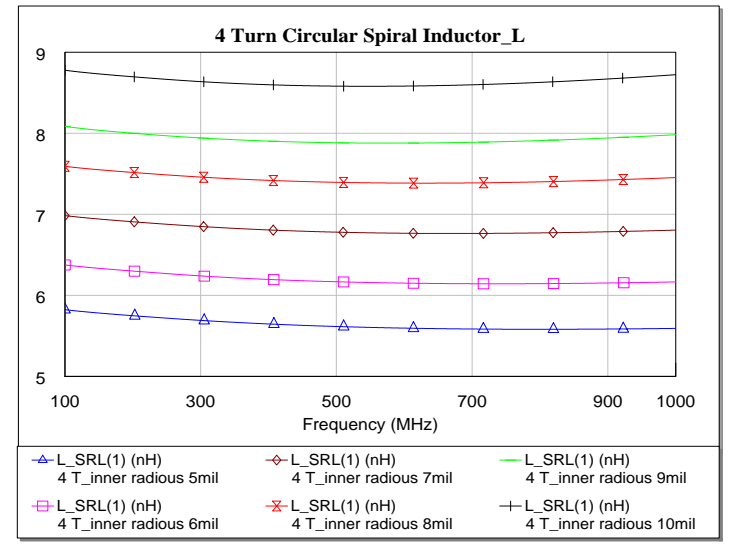

Fig-9:4 TurnsCircular Spiral Inductor (L)

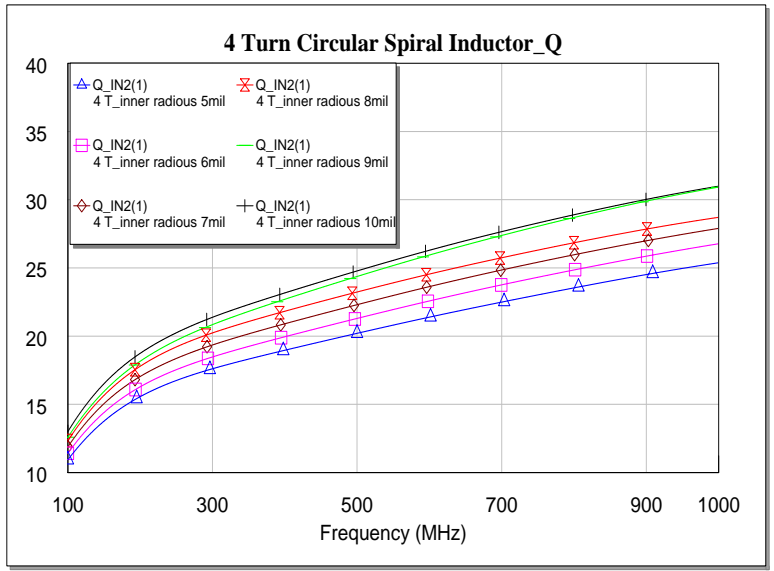

Fig-10:4 TurnsCircular Spiral Inductor (QF)

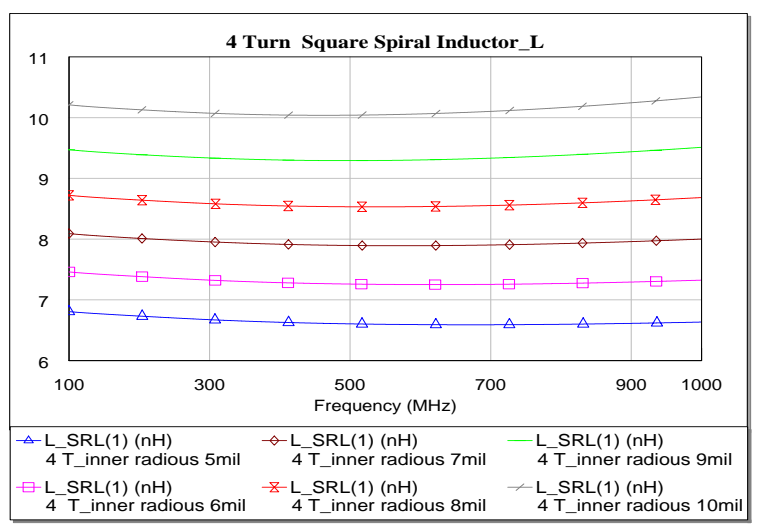

Fig-11:4 TurnsSquare Spiral Inductor (L)

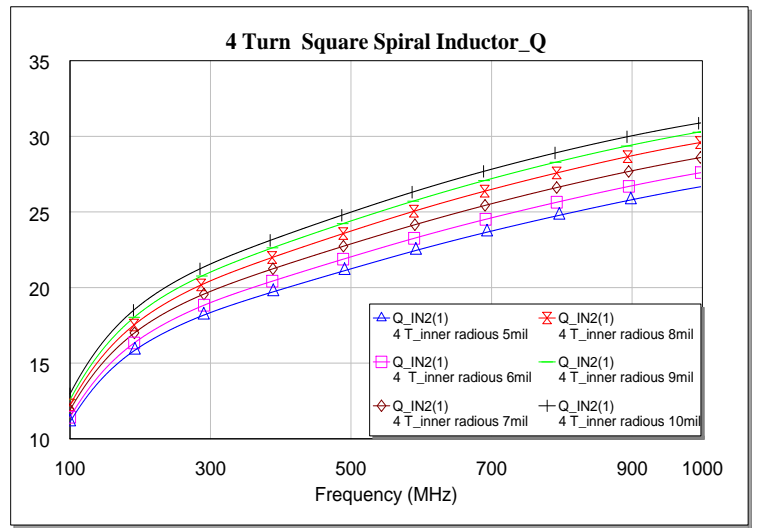

Fig-12:4 TurnsSquare Spiral Inductor (QF) 


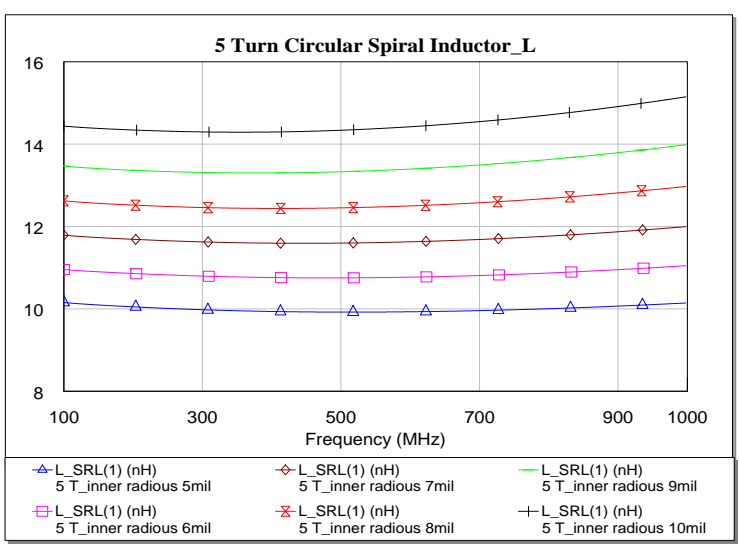

Fig-13:5 TurnsCircular Spiral Inductor (L)

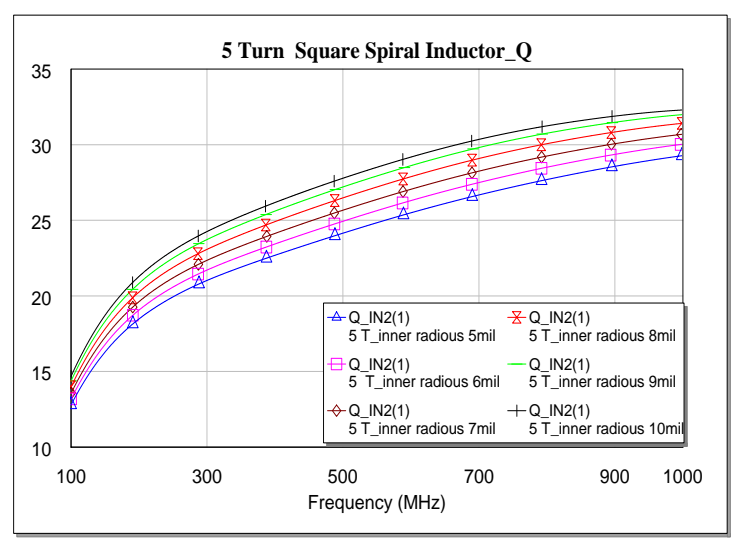

Fig-14:5 TurnsCircular Spiral Inductor (QF)

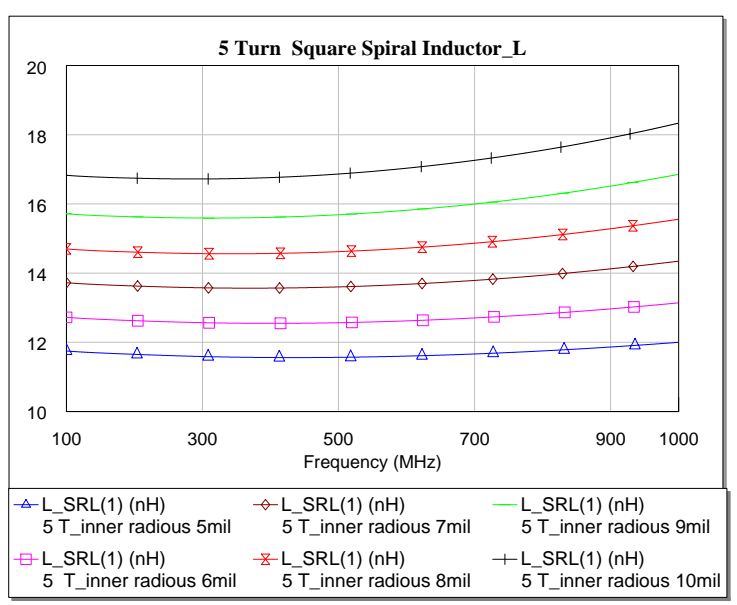

Fig-15:5 TurnsSquare Spiral Inductor (L)

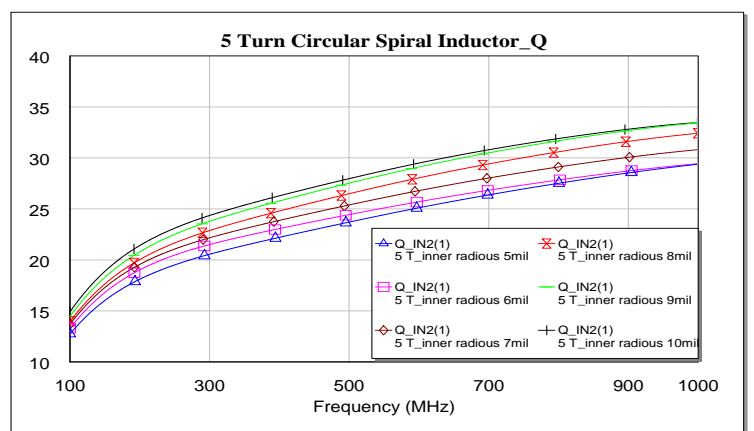

Fig-16:5 TurnsSquare Spiral Inductor (QF)

The plotted inductor graphs shows that increasing inner diameter of spiral inductor will affect increases inductance value. The inner diameter is directly related to outer diameter of the spiral inductor. The radius of an inner diameter is immense to increases the passive component effect due to closed edges of the spiral inductor. To change the inner diameter of the spiral inductor set for desired inductance value. The plotted graphs of quality factor versus frequency shows that increase the inner radius will effect to increase quality factor of the spiral inductor.

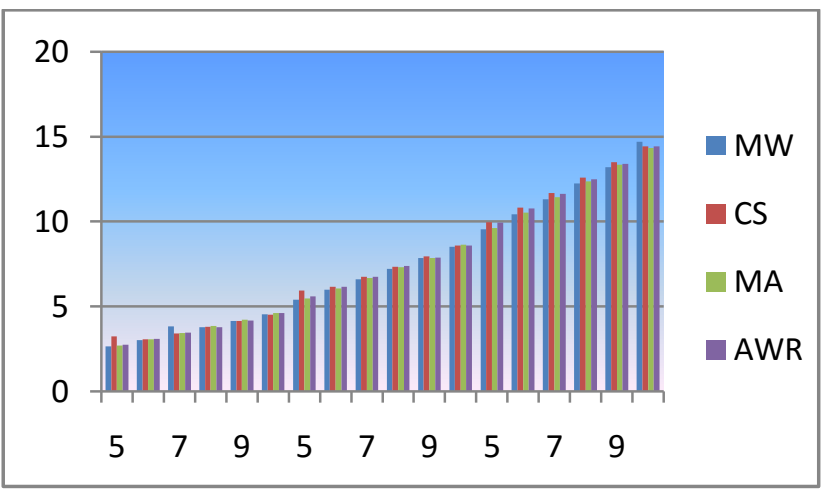

Fig-17:The Circular Spiral Inductance V/S Inner Diameter

The figure 17 and 18 shows that the comparison of electromagnetic field simulated circular and square spiral inductor with MW, CSA and ME simple equations. The observed results shows that electromagnetic field simulated results has good agreement with each other. The average accuracy for comparison is more than $98 \%$.

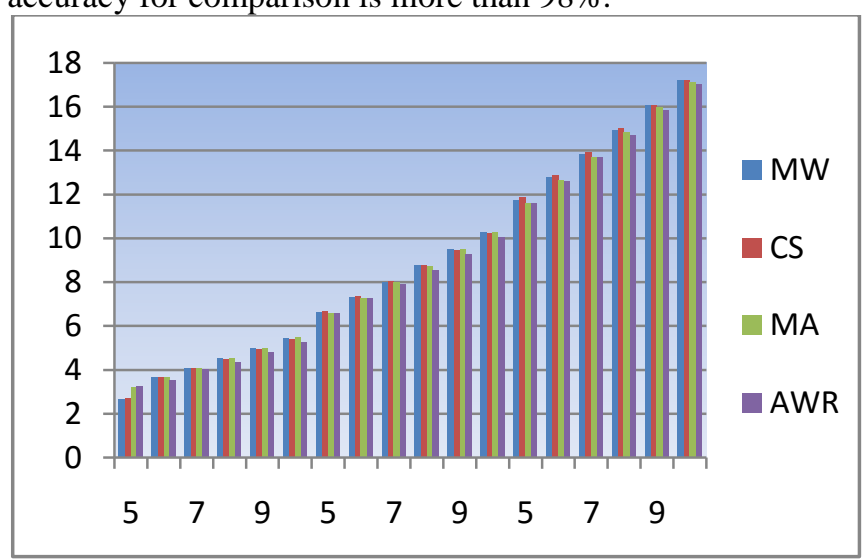

Fig-18:The Square Inductance V/S Inner Diameter 


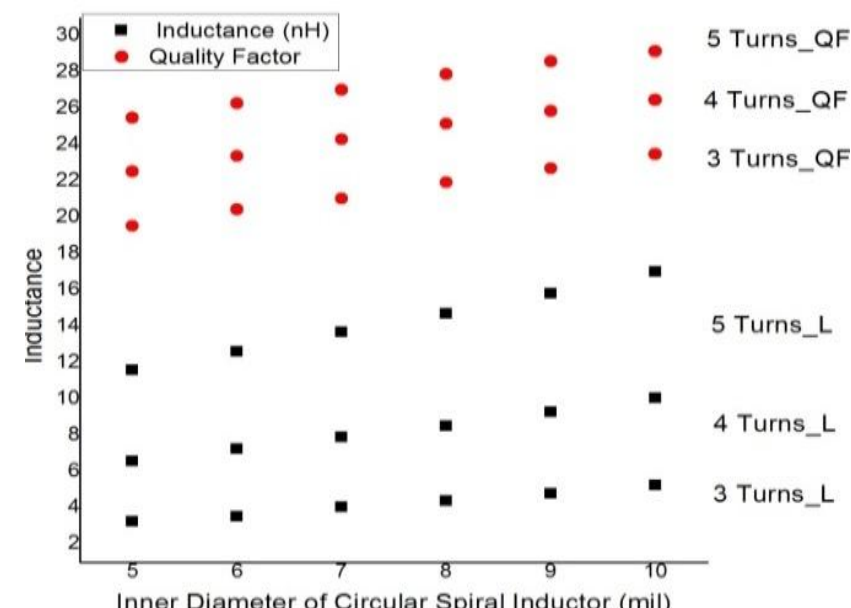

Fig-19:The Circular Spiral Inductance v/s Inner dDiameter with Turns

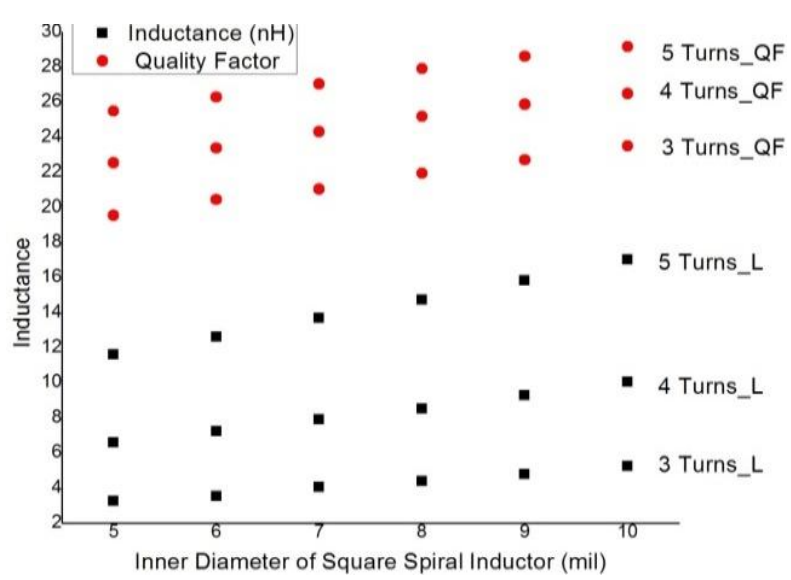

Fig-20:The Square Spiral Inductance v/s Inner dDiameter with turns

The figure 19 and 20 shows circular and square spiral inductance versus inner diameter with respect to number of turns. The results displays that increase the inner diameter and turns will effect to increase the surface area will affect to decreases quality factor of the spiral inductor [31]. The accuracy of circular simulated design is better than square shows in figure 19 and 20 . The quality factor is a figure merit in spiral inductor and it can be achieve more in circular spiral inductor than square.

\section{CONCLUSION}

The circular and square spiral inductors are designed using basic relations. The obtained electromagnetic field simulated results arecomparing with standard simple equations like MW, CSA and ME. The observed results are well match with electromagnetic field simulated results using NI/AWR tool. The validation of spiral inductor is done with different inner diameter (5mil to $10 \mathrm{mil}$ ) and number of turns (3turns to 5 turns) of the spiral inductor.The analysis is done using scattering parameter technique and the average comparison accuracy is more than $98 \%$ is achieved for spiral inductance design. The designed operating frequency of $600 \mathrm{MHz}$ and attain quality factor with respect to simulated results. This study is much useful to implementing in RF/Microwave circuit development.

\section{ACKNOWLEDGMENT}

The authors would like to thank ICON design Automation Pvt Ltd Bangalore, for their help and guidance to carry out this work at their R\&D lab.

\section{REFERENCE}

[1]. C. P.Yueet al., "A physical model for planar spiral inductors on silicon," IEDM Tech. Dig., pp. 155158, Dec. 1996.

[2]. R. D. Lutz et al., "Modeling of spiral inductors on lossy substrates for RFIC applications," Proc. 1998 Radio Frequency Integrated Circuits Symp., pp. 313316, June 1998.

[3]. J. Crols, P. Kinget, J. Craninckx, and M. S. J. Steyaert, "An analytical model of planar inductors on lowly doped silicon substrates for high frequency analog design up to $3 \mathrm{GHz}$," in 1996 Symp. VLSI Circuits Dig. Tech. Papers, pp. 28-29, June 1996.

[4]. T. H. Lee and S. S. Wong, CMOS RF integrated circuits at $5 \mathrm{GHz}$ and beyond, Proc. IEEE, Vol. 88, pp. 1560-1571, Oct. 2000.

[5]. T. H. Lee, The Design of CMOS Radio-Frequency Integrated Circuits, Cambridge University Press, 2002.

[6]. Jingxue Lu, Fengyi Huang, Yusong Chi, An Analytical Approach to Parameter Extraction for OnChip Spiral Inductors With Double- $\pi$ Equivalent Circuit, IEEE Radio Frequency Integrated Circuits (RFIC) Symposium, (2006).

[7]. Myounggon Kang, Joonho Gil and Hyungcheol Shin, A Simple Parameter Extraction Method of Spiral OnChip Inductors, IEEE Trans. on Electron Devices, vol. 52, n. 9,( 2005).

[8]. Joonho Gil and Hyungcheol Shin, A Simple WideBand On-Chip Inductor Model for Silicon-Based RF ICs, IEEE Trans. on Microwave Theory and Techniques, vol. 51, n. 9, (2003).

[9]. Ivan C. H. Lai and Minoru Fujishima, A New OnChip Substrate-Coupled Inductor Model Implemented With Scalable Expressions, IEEE Journal of Solid-State Circuits, vol. 41, n. 11, (2006).

[10]. Fengyi Huang, Jingxue Lu, Nan Jiang, Xiaowen Zhang, Wengang $\mathrm{Wu}$, and Yangyuan Wang, Frequency-Independent Asymmetric Double- $\pi$ Equivalent Circuit for On-Chip Spiral Inductors: Physics-Based Modeling and Parameter Extraction, IEEE Journal of Solid-State Circuits, vol. 41, n. 10, (2006).

[11]. N. R. Das and AMitra, A New Approach to The Modeling of Si-RFIC Inductor, Microwave and Optical Technology Letters, vol. 48, n. 6, (2006).

[12]. Heng-Ming Hsu, Effective Series-Resistance Model of Spiral Inductors, Microwave and Optical Technology Letters, vol. 46, n. 2, (2005).

[13]. Pedder, D.J.: Technology and infrastructure for embedded passive components. On Board Technology, pp. 8- 11 (2005). 
[14]. Ulrich, R.K., Brown, W.D., Ang, S.S., Barlow, F.D., Elshabini, A., Lenihan, T.G., Naseem, H.A., Nelms, D.M., Parkerson, J., Schaper, L.W., Morcan, G.: Getting aggressive with passive devices. IEEE Circuits Devices Mag. 16(5), 16-25 (2000).

[15]. Jinglin Shi and et al, The Enhancement of Q Factor for Patterned Ground Shield Inductors at High Temperatures, IEEE Trans. on Magnetics, vol. 42, n. 7, (2006).

[16]. Ban-Leong Ooi, Dao-Xian Xu, Detailed Analysis of High-Quality Symmetrical Octagonal Spiral Inductors on Si Substrate, Published online in Wiley Inter Science, (2005).

[17]. Chang-Lee Chen, Effects of CMOS Process Fill Patterns on Spiral Inductors, Microwave and Optical Technology Letters, vol. 36, n. 6, (2003).

[18]. Pan, S. J., L. W. Li, and W. Y. Yin, "Performance trends of on-chip spiral inductors for RFICs," Progress in Electromagnetic Research, Vol. 45, 123151, 2004.

[19]. C. Patrick Yue and S. Simon Wong, "Physical Modeling of Spiral Inductors on Silicon," March 2000, IEEE Transaction on Electron Devices, vol. 47, no. 3 , pp. $560-568$.

[20]. F. W. Grover, Inductance Calculations. 1962, New York, NY: Van Nostrand.

[21]. SiamakSalimy, Antoine Goullet, Ahmed Rhallabi, FatihaChallali, Serge Toutain, Jean Claude Saubat, "A Unified Analytical and Scalable Lumped Model of RF CMOS Spiral Inductors Based on Electromagnetic Effects and Circuit Analysis," Elsevier, Solid state electronics, 2011.

[22]. C. P. Yue, C. Ryu, J. Lau, T. H. Lee, and S. S. Wong, "A physical model for planar spiral inductors on silicon," in Proc. IEEE Int. Electron Devices Meeting, San Francisco, CA, 1996.

[23]. R. B. Merrill, T. W. Lee, H. You, R. Rasmussen, and L. AS. Moberly, "Optimization of high Q integrated inductors for multilevel metal CMOS," in Proc. IEEE Int. Electron Devices Meeting, Washington, DC, 1995, pp. 38.7.1-38.7.4.

[24]. Ivan C. H. Lai and Minoru Fujishima, A New OnChip Substrate-Coupled Inductor Model Implemented With Scalable Expressions, IEEE Journal of Solid-State Circuits, vol. 41, n. 11, (2006).

[25]. J. Bahl, "High-performance inductors," IEEE Trans. Microwave Theory Tech., vol. 49,no. 4,pp. 654664,Apr. 2001.

[26]. Sunderarajan S. Mohan, Maria del Mar Hershenson, Stephen P. Boyd, and Thomas H. Lee, "Simple Accurate Expressions for Planar Spiral Inductances," IEEE Journal of Solid-State Circuits, Vol. 34, No. 10, Oct 19991419.

[27]. H. A. Wheeler, "Simple inductance formulas for radio coils," in Proc. IRE, Oct. 1928, vol. 16, no. 10, pp. 1398-1400.

[28]. E. B. Rosa, "Calculation of the self-inductances of single-layer coils," Bull. Bureau Standards, vol. 2, no. 2, pp. 161-187, 1906.
[29]. M. Hershenson, S. S. Mohan, S. P. Boyd, and T. H. Lee, "Optimization of inductor circuits via geometric programming," in Design Automation Conf., New Orleans, LA, June 1999, pp. 994-998.

[30]. J A Power, S C Kelly, E C Griffith and M O Nell, "An Investigation of On-Chip Spiral Inductor on a $0.6 \mu \mathrm{m}$ BiCMOS Technology for RF Applications," Proc, IEEE, Microelectronic Text Structures, Vol 12, March 1999. 